1 Title: Mapping the sex determination region in the Salix $F_{1}$ hybrid common parent population

2 confirms a ZW system in six diverse species

4 Authors: Dustin G. Wilkerson ${ }^{1}$, Bircan Taskiran ${ }^{1,2}$, Craig H. Carlson ${ }^{1,3}$, and Lawrence B. Smart ${ }^{1}$

6 Affiliations: ${ }^{1}$ Horticulture Section, School of Integrative Plant Sciences, Cornell University,

7 Cornell AgriTech, Geneva, NY 14456

8 Current Affiliations: ${ }^{2}$ Biology Department, Faculty of Science, Cankiri Karatekin University,

9 Cankiri, Turkey; ${ }^{3}$ USDA-ARS, Cereal Crops Research Unit, Edward T. Schafer Agricultural

Research Center, Fargo, ND 58102

12 Short Running Title: ZW SDR in Salix $F_{1}$ families

14 Key Words: QTL Mapping; Salix; sex determination; linkage map; Salix purpurea; Salix viminalis;

15 Salix suchowensis

16

17 Corresponding Author: Lawrence Smart; 630 W. North Street, 111 Surge Lab, Geneva, NY 
Within the genus Salix, there are approximately 350 species native primarily to the mine novel alleles conferring variation important for production as a bioenergy crop, but also to

24 identify evolutionarily important genes, such as those involved in sex determination. To

25 leverage this diversity, we created a mapping population by crossing six Salix species (S. viminalis, S. suchowensis, S. integra, S. koriyanagi, S. udensis, and S. alberti) to common male

27 and female S. purpurea parents. Each family was genotyped via genotyping-by-sequencing and assessed for kinship and population structure as well as the construction of 16 backcross linkage maps to be used as a genetic resource for breeding and selection. Analyses of population structure resolved both the parents and $F_{1}$ progeny to their respective phylogenetic

31 section and indicated that the S. alberti parent was misidentified and was most likely $S$. suchowensis. Sex determining regions were identified on Salix chromosome 15 in the femaleinformative maps for seven of the eight families indicating that these species share a common

34 female heterogametic ZW sex system. The eighth family, S. integra $\times$ S. purpurea, was entirely

35 female and had a truncated chromosome 15. Beyond sex determination, the Salix $F_{1}$ hybrid

37 genetic factors underlying complex traits, aid in marker-assisted selection, and support genome assemblies for this promising bioenergy crop. 
The establishment of genomic resources is an important step in developing a fully

41

42

43

44

realized breeding program, reinforced by modern tools for trait mapping, candidate gene identification, and marker-assisted selection. Salix and Populus (poplars) comprise the majority of species in the family Salicaceae, which consists of dioecious trees, shrubs, and subshrubs that are highly heterozygous. Shrub willow (Salix spp.) are grown in northern latitudes as a sustainable, high-yielding, carbon neutral, bioenergy crop that can grow on marginal land and provide multiple ecosystem services (Smart et al. 2005; Stoof et al. 2014; Clifton-Brown et al. 2019; Fabio and Smart 2020). While shrub willow breeding in the United States has been active since the 1980 's, most of the nearly 350 species have yet to be tapped as a source of genetic diversity (Dickmann and Kuzovkina 2014; Stanton et al. 2014).

Genomic resources developed for poplar were used in early genomic studies in Salix, because their genomes are largely co-linear (Hanley et al. 2006; Berlin et al. 2010). As sequencing technologies became increasingly more affordable, more Salix genome sequencing was completed and now there are several high-quality assemblies. Genomic resources for Salix are currently centered around a few key species. In Europe, Salix viminalis is an important bioenergy crop species with a recently published, high quality genome assembly (Almeida et al. 2020). S. viminalis has been used in several QTL mapping studies for resistance to willow leaf rust (Melampsora larici-epitea) (Rönnberg-Wästljung et al. 2008; Samils et al. 2011; Sulima et al. 2017), drought tolerance (Rönnberg-Wästljung et al. 2005), and growth and phenology (Hallingbäck et al. 2016; Hallingbäck et al. 2019). While in the United States, Salix purpurea is the model species for bioenergy willow breeding, genetics, and genomics. The US Department of Energy Joint Genome Institute has produced the highest quality annotated Salix reference 
62

genomes assembled in the genus on male and female S. purpurea, available on Phytozome

(Zhou et al. 2018; Zhou et al. 2020) (https://phytozome-

next.jgi.doe.gov/info/SpurpureaFishCreek v3 1 ; https://phytozome-

next.jgi.doe.gov/info/Spurpurea_v5 1). Using joint linkage and association mapping

approaches focused on S. purpurea, Carlson et al. (2019) identified numerous QTL for a wide

range of morphological, physiological, insect and disease resistance and biomass composition

traits. A naturalized species in North America, S. purpurea is a potential donor of broad

adaptability traits for species susceptible to pests and diseases in the Northeast United States.

There is value in studying the genomes of less-characterized Salix species for phylogenomic

analysis and to discover diverse sources of alleles for introgression into elite yielding cultivars.

Here, we introduce the Salix $F_{1}$ hybrid common parent population (Salix $F_{1} H C P$ ). The

parents, described in Fabio et al. (2019) and Crowell et al. (2020), represent a diverse selection

of species from Salix subgenus Vetrix (Dickmann and Kuzovkina 2014). Six Salix species (S.

viminalis, S. suchowensis, S. integra, S. koriyanagi, S. udensis, and S. alberti) were crossed to

common male and female S. purpurea parents to form eight species hybrid families. Literature

describing these species ranges from the high-quality reference genomes available for $S$.

purpurea and S. viminalis to the scarcely studied S. alberti (Rosso et al. 2013). Salix suchowensis

is native to China and has been used recently to generate a chromosome scale genome

assembly (Wei et al. 2020). This species has been assessed for its response to drought stress (Jia

et al. 2020) and was one of the first Salix species used to map the sex determination region

(SDR) (Liu et al. 2013). Salix udensis, formally known as S. sachalinensis, has been described as a

Japanese riparian willow species that acts as a natural nest cavity for fish owls (Niiyama 2008; 
Slaght et al. 2018) and is suggested to have sexually dimorphic characteristics (Ueno and Seiwa 2003; Ueno et al. 2006). Some genomic resources are available for the Korean S. koriyanagi, as its chloroplast genome have been sequenced (Kim et al. 2019; Park et al. 2019) while S. integra has been assessed for its phytoremediation potential (Cao et al. 2020; Yang et al. 2020b). Developed to interrogate the genetics of several understudied Salix species, the Salix $F_{1}$ HCP is an important step in the development of genomic resources in Salix.

The Salicaceae represents an interesting family for the study of the evolution of dioecy and the mechanisms of sex determination. Genetic mapping of SDRs in the Salicaceae have revealed considerable intra- and inter-chromosomal variability and contrasting sex determination systems (Yang et al. 2021). While $P$. alba and $P$. trichocarpa are male heterogametic (XY) with SDRs located on chr19, the SDR of $P$. euphratica was identified on chr14 and is female heterogametic (ZW) (Paolucci et al. 2010; Geraldes et al. 2015; Yang et al. 2021). Conversely, the SDR of S. purpurea has been mapped to a large, pericentromeric region on Salix chr15, but has been found to maintain a few orthologous regions present within the $P$. trichocarpa chr19 SDR (Zhou et al. 2018). However, it is unclear whether dioecy evolved before or after their divergence (Hou et al. 2015). The sex systems of Salix also lack a consensus model. For instance, similar to S. purpurea, both S. suchowensis (Hou et al. 2015; Chen et al. 2016) and S. viminalis (Pucholt et al. 2015; Pucholt et al. 2017b) have female heterogametic (ZW) systems with SDRs located on Salix chr15, yet tree-form S. nigra has an XY system SDR on chr07 (Sanderson et al. 2021). Considering the variability in sex determination systems and SDR locations already discovered within Salix, elucidating the mechanisms of sex determination in more species could help to build a more cohesive understanding of SDR evolution. Further 
investigation could provide evidence to implicate SDR turnovers as a factor in the considerable species diversity within the genus.

To establish the Salix $\mathrm{F}_{1}$ HCP as a genetic resource, this study sought to: (1) describe the genetic relationships between the parent species, (2) develop female and male parent informative linkage maps for each family, and (3) define and compare their respective SDRs.

MATERIALS AND METHODS

\section{Population Development}

Females of S. viminalis, S. integra, S. alberti, and S. suchowensis were crossed with male

S. purpurea clone ID 94001, while males of S. viminalis, S. udensis, S. koriyanagi, and S. suchowensis were crossed with female S. purpurea clone ID 94006 creating eight $F_{1}$ species hybrid families (Fig. 1). The common S. purpurea parents, 94006 and 94001, were chosen based upon their differential resistance to willow leaf rust (Crowell et al. 2020), adaptation to the Northeastern U.S., and availability of high-quality reference genomes (Zhou et al. 2020).

Crosses were made by forcing floral catkins of the parent genotypes from dormant shoots in a greenhouse. All crosses were made in isolation to prevent pollen contamination. When anthers began to dehisce, male catkins were excised and placed in falcon tubes for pollen extraction using toluene, as described in Kopp et al. (2002), then stored in $2 \mathrm{~mL}$ microcentrifuge tubes at $-20^{\circ} \mathrm{C}$ until female catkins were receptive. Seedlings were established in a standard peat-based potting mix in a greenhouse, then transplanted to nursery beds near Cornell AgriTech (Geneva, NY). Winter dormant cuttings from all parents and progeny were collected from one-year old stems and hand-planted in the field in a randomized complete block design 
127 with three plants per plot and four replicate blocks. Field trials were established with $1.83 \mathrm{~m}$

128 spacing between rows and $40.6 \mathrm{~cm}$ between plants within rows.

DNA Extraction and Genotyping-by-Sequencing

Kit (QIAGEN Inc., Valencia, CA, USA). After checking the DNA quality using gel electrophoresis,

100 bp) on the Illumina HiSeq 2500 (Illumina, Inc., San Diego, CA, USA) platform.

143 (Zhou et al. 2020). As this genome includes both chr15Z and 15W, chr15Z was excluded to

144 reduce mapping errors within the pseudoautosomal regions surrounding the SDR in S.

145 purpurea. This process was repeated once for all eight families together and then for each of

146 the eight $F_{1}$ families separately to identify population-wide and family-specific SNPs. By calling

147 variants on all eight families and then on each family separately, variants were based on inter- 
148

149

150

151

152

153

154

155

156

157

and intra- familial genetic differences, respectively. The resulting VCF files contained 684,412

SNPs for the full analysis and ranged from 174,762 to 266,797 SNPs depending on the family.

On the eight $F_{1}$, SNPs with $>70 \%$ missing data and minor allele frequency $<0.01$ and $F_{1}$

individuals were dropped if they had $>80 \%$ missing data or determined to be outliers based on

principal component analysis. Missing genotype calls and low read depth are common in GBS

(Elshire et al. 2011), therefore imputation was performed separately on each family to validate

calls in haplotype blocks. Using LinkImputeR (Money et al. 2017), genotypes called with a read

depth $<5$ were set to missing before filtering again for missingness $>70 \%$, resulting in 95,281 to

145,944 imputed SNPs with accuracies ranging from 84.3 to $93.1 \%$.

\section{Population Structure}

SNPs were filtered to retain markers and individuals with $\leq 20 \%$ missing data and SNPS

with a minor allele frequency $>0.01$, which resulted in 55,398 SNPs. Analysis of principal

components and kinship using default parameters were performed in Tassel 5 and visualized in

$R$ ( $R$ Core Team 2020). Multiple runs of the parents and 10 randomly selected $F_{1}$ progeny were analyzed using fastSTRUCTURE (Raj et al. 2014). Only a subset of the $F_{1}$ were used in this analysis in order to manage file size and computation requirements. Multiple analyses of 'structure.py' were completed ( $K=3$ - 10). Using 'chooseK.py', $K=6$ represented the model complexity that maximized the marginal likelihood and best explained the data structure, suggesting six separate populations.

\section{Linkage Map Construction and Analysis}


Unless otherwise indicated, linkage map construction and analysis were performed

172 the analysis. If only one parent was set to missing, its genotype was inferred based on the

173 genotype of the known parent and the segregation of the $F_{1}$. These parental consensus

174 genotypes were used to identify the female informative $(A B \times A A)$ and male informative $(A A \times$

$175 \mathrm{AB}$ ) markers used to generate backcross linkage maps for each parent using a combination of

176 R/qtl (Broman et al. 2003) and ASMap (Taylor and Butler 2017). Co-located markers and those

177 exhibiting extreme segregation distortion were removed using ASMap function 'pullCross'.

178 Linkage groups were then created using 'mstmap' with default parameters except for

179 objective.fun = "ML" and bychr = FALSE. The $\mathrm{p}$-value for determining linkage groups varied

180 between families, ranging from 1e-6 to 1e-12, depending on the demarcation of the 19

181 expected linkage groups. A custom R function was then used to perform simple error correction

182 to reduce the number of double crossovers and deflate map distances before reforming linkage

183 groups. Briefly, this function relies on the marker order determined after formation of linkage

184 groups, identifying double crossovers of $\leq$ two SNPs, and correcting them. Map quality was

185 checked using two strategies. The 'heatMap' function in ASMap, which plots LOD linkage

186 between markers on the upper triangle and estimated recombination frequency on the bottom,

187 reveals markers that are problematic or out of phase. Then by comparing the physical position

$188(\mathrm{Mb})$ based on alignment to the $S$. purpurea reference genome and the genetic distance (cM) 
within each linkage group shows issues with marker order or potential chromosomal rearrangements.

To delimit the SDR in each family, the sex of all $F_{1}$ individuals was recorded by inspecting flowering catkins over two growing seasons in the field. Sex ratio bias was tested using a chisquare test for a 1:1 sex ratio. Using functions in R/qtl, genotype probabilities were calculated using 'calc.genoprob' (step =0, error.prob $=0.01$, map.function $=$ "kosambi", stepwidth $=$ "fixed"). Next, QTL mapping for individual sex was performed using 'scanone': model = "binary", method = "em". Genome-wide significance thresholds were determined based on the results of 'scanone.perm' (n.perm = 1000). QTL positions were refined using 'refineqtl' (method $=$ "hk", model = "binary"), then the 1.5 LOD support intervals were calculated using 'lodint' with default parameters.

\section{RESULTS}

\section{Population Structure}

A combination of PCA, hierarchical clustering, and fastSTRUCTURE were used to describe the population structure of the Salix $\mathrm{F}_{1} \mathrm{HCP}$. A PCA of the parents revealed three distinct clusters, formed by two PCs accounting for $36.8 \%$ and $22.5 \%$ of the total genetic variation (Fig. 2A). The two common S. purpurea parents, 94006 and 94001, formed a single group and were separated from the other parents by PC2. The S. viminalis parents, 'Jorr' and 07-MBG-5027, formed a cluster with S. udensis 04-BN-051, which was differentiated from the remaining species by $\mathrm{PC} 1$. Including the $\mathrm{F}_{1}$ progeny from each family into the analysis, each PC accounted for $26 \%$ and $10.1 \%$ of the total variation (Fig. 2B). PC2 split S. udensis from the two S. 
viminalis parents and separated S. koriyanagi from S. suchowensis, S. integra, and S. alberti. As

211

212

expected, the $F_{1}$ individuals were intermediate between the common parent and family specific species. The $F_{1}$ progeny derived from the female parents S. suchowensis P295, S. alberti P294, and S. integra P336 (all crossed with male S. purpurea 94001), co-localized.

Kinship analysis grouped the two S. viminalis parents with S. udensis, the two $S$. purpurea parents together, and S. koriyanagi with S. integra (Fig. 2C). Salix suchowensis and the S. viminalis and S. udensis group were the least similar while S. purpurea grouped centrally between them, similar to PC1 (Fig. 2A). Within S. suchowensis, the female P295 was more closely related to the female S. alberti P294 than it was to P63, a male. Admixture analysis included the parents and a subset of the $F_{1}$ individuals of each family. The eight groups of $F_{1}$ individuals were comprised of roughly half the genetic background of S. purpurea and half the other species parent, as expected (Fig. 2D). Salix viminalis, S. integra, S. koriyanagi, S. udensis and S. purpurea formed distinct clusters, while S. alberti P294 grouped together with S. suchowensis.

\section{Linkage Map Construction and Analysis}

Since there is considerable divergence between parents and pedigrees, variant discovery and marker filtration were performed for each family separately. Using consensus genotypes derived from multiple sequencing runs of the parents, markers were split into female $(A B \times A A)$ and male $(A A \times A B)$ informative backcross markers for linkage map construction, which resulted in 16 linkage maps (Fig. 3). Each linkage map consisted of 19 linkage groups with total map lengths ranging from 3939.9 - 6957.3 cM containing between 
2035 and 3852 total markers (Table S1). Recombination frequency and genetic to physical on a simple chi-square test $(p<0.05)$, with female to male ratios ranging from 1.4 to 1.6 (Table family was entirely female. Single QTL for sex were identified on chr15 within seven of the eight maternal maps, excluding S. integra of $13 X-426$, explaining between $60.7-74.1 \%$ of the total phenotypic variation (Table 2). LOD scores for the female and male linkage maps are in Table S2 and Table S3, respectively. Genome-wide LOD significance thresholds ranged from 3.39 - 3.69, depending on the map. On the four maternal S. purpurea maps, the QTL as determined by the permuted significance thresholds accounted for $10.3-12.03 \mathrm{Mb}$ of the roughly $15.5 \mathrm{Mb}$ chr15 due to suppressed recombination (Figure 4). QTL refinement and 1.5 LOD support intervals narrowed this down to account for $1.17-7.31 \mathrm{Mb}$ with peak markers located at 2.91, 2.92, 7.33, and 8.65 Mb when S. suchowensis, S. viminalis, S. koriyanagi, and S. udensis were the paternal parent, respectively (Table 2; Figure 4). Of the three remaining maternal maps, QTL

247 determined by LOD significance accounted for 12.3, 11.45, and $13.55 \mathrm{Mb}$ of chr15 on the $S$. alberti, S. suchowensis, and S. viminalis maps, respectively, reflecting suppressed 
Salix is a very diverse genus, consisting of more than 350 species. As the genomic tools the ability to characterize and deploy Salix's innate diversity in improving high yielding cultivars.

255 That diversity extends to variation between species even in traits as evolutionarily important as sex determination (Yang et al. 2021). By generating mapping populations that include

257 characterized species crossed with those less studied, we will increase the number of Salix 258 species available for trait introgression. We developed the Salix $F_{1} H C P$ as a resource to characterize the interactions between alleles from different, but related species, and to map variation in important traits. Using GBS, we analyzed the population structure among the eight

261 families, generated linkage maps of each of the parents using backcross markers and mapped

262 the SDR in seven of the eight families using phenotypes collected from repeated field surveys.

263 PCA and hierarchical clustering predominately resolved the population by section with the $F_{1}$

264 clustering between the parents as expected. In each analysis, S. alberti P294 was found to very

265 closely related to the S. suchowensis parents. Given these results and the limited publicly 266 available information about S. alberti, P294 is likely S. suchowensis and will be described as 267 such.

Among the 16 linkage maps produced, all QTL for sex were detected on chr15 in seven

269 of the eight families and only in the maternal maps. In a recent study mapping sex in S. triandra 270 using backcross markers, Li et al. (2020) were also only able to detect QTL within the maternal

271 map, which is indicative of a ZW sex determination system on chr15. While this had been

272 known for S. purpurea (Zhou et al. 2018), S. viminalis (Pucholt et al. 2017b), and S. suchowensis

273 (Chen et al. 2016), this is the first study to report that both S. koriyanagi and S. udensis have ZW 
SDR on chr15. Although the location of the SDR has also not been reported in S. integra, the S.

275

276

277

278

279

280

281

282

283

284

285

286

287

288

289

290

291

292

293

294

integra $\times$ S. purpurea family was entirely female and therefore was excluded in the linkage analysis. The chr15 map from S. integra P336 is considerably smaller than the other families, aligning to only the $14.2-15.5 \mathrm{Mb}$ region of the $S$. purpurea reference genome, while maps of from of the other families had near complete coverage. The region S. integra P336 chr15 that did have segregating markers was outside the SDR intervals of the other female maps and is likely in the pseudoautosomal region of the sex chromosome.

Six of the eight families were female biased - a feature prevalent in Salix. Of those studied here, S. purpurea (Gouker et al. 2020), S. viminalis (Alström-Rapaport et al. 1997), S. suchowensis (Yang et al. 2020a), S. udensis (Ueno and Seiwa 2003), and S. integra (Tozawa et al. 2009) have documented cases of sex ratio bias, yet this is the first time it has been reported in S. koriyanagi. The genetic basis of sex ratio bias could be a result of secondary sex dimorphisms, such as higher mortality rates in males, increased herbivory and pathogen resistance in females, or the presence of a sex distorter locus (Pucholt et al. 2017a).

The mapping of the SDR in this study resulted in marker associations that represented a majority of chr15 prior to refinement. Of the four families with S. purpurea as the common female parent, refined QTL shared similar ranges of the SDR described by Zhou et al. (2020) (6.8 $\mathrm{Mb}$ in length starting at $2.3 \mathrm{Mb}$ ) with the exception of the S. purpurea $\times$ S. viminalis family (1.17 Mb). Carlson et al. (2019) mapped the SDR to $4.5-11.4 \mathrm{Mb}$ on chr15 using a S. purpurea $\mathrm{F}_{2}$ population. This study localized the SDR to the same pericentromeric region of chr15 in S. purpurea using $\mathrm{F}_{1}$ hybrid families. 
The most recent delimitation of the SDR in S. viminalis spanned roughly $3.4 \mathrm{Mb}$ (approx.

2.75 $\mathrm{Mb}$, including the position of our peak marker, even though we aligned markers to a different reference genome. Almeida et al. (2020) aligned the SDR map to chr15 of both S. purpurea v.1.0 and S. viminalis reference genomes and found overall synteny between species, yet with several structural rearrangements. This contrasts with what is seen in our results, where the chr15 of 07-MBG-5027 showed only minor rearrangements, likely due to alignment to the high-quality S. purpurea v5.1 reference genome.

Based on annotations from the Populus trichocarpa genome, differential gene expression analysis in S. suchowensis between male and female plants led to predictions that the SDR was originally on chr14 (Liu et al. 2013). However, later work repositioned the SDR to the centromeric region of chr15 when based on Salix alignment (Chen et al. 2016). Our study defined the physical distance of the SDR at 2.44-9.35 Mb in P295 and 8.63-10.22 Mb in P294 with an overlap of $0.72 \mathrm{Mb}$. These two linkage maps show the greatest amount of rearrangement on chr15 when aligned to the $S$. purpurea reference. It is fair to conclude that alignment to a future S. suchowensis reference genome would aid in improving the mapping resolution in this region.

313 the map of chr15 from each family, the SDR extends across a region of sparce marker density, 314 approximately 3 to $9 \mathrm{Mb}$ and is flanked by regions with greater marker density. As described above, this centromeric region with suppressed recombination is often associated with the SDR 316 in Salix. In all seven families with mapped SDR, the peak marker was located within this region 
317 although its position varied. In the two S. viminalis families and two of the three S. suchowensis

318 families, the peak marker was located within a $0.38 \mathrm{Mb}$ region (2.57 $-2.95 \mathrm{Mb}$ ), while the

319 remaining three families were less consistent and located proximal to the centromere. The

320 generation of additional reference genomes for use in mapping will add context to these results

321 and further refine the structure of the SDR among variable species.

This study described the population structure among the eight families within the Salix

$323 \mathrm{~F}_{1} \mathrm{HCP}$, constructed linkage maps for each parent, and mapped the SDR to the maternal chr15

324 in seven of the eight families. The introduction of the Salix $F_{1}$ HCP provides the opportunity to

325 map QTL for phenotypic traits beyond sex determination, while the linkage maps could be used

326 to anchor and scaffold contigs in the generation of new reference genomes for each of the

327 parents. While all species have a ZW sex determination system with an SDR that maps to chr15,

328 these genetic resources provide a foundation for further characterization of the mechanism of

329 sex determination and mapping of other key traits in these related species.

\section{Data Availability}

The GBS data used in the population structure analysis and the eight files used for

332 linkage map construction each family prior to linkage map construction are available in hapmap

333 format through figshare at https://figshare.com at "GBS Data from Wilkerson et al". R code

334 used to format and create the linkage maps is available on the Willowpedia Github site located

335 at https://github.com/Willowpedia/Wilkerson_etal_SalixLinkageMaps. Table S1 contains

336 statistics on each of the 16 linkage maps created in this study, including marker count and cM

337 length for each linkage group and the total markers and cM length for each map. Figure S1 is a 
pdf slide show that, one map per slide, shows the marker cM by physical position for each

linkage group and a heatmap visualizing recombination frequency and linkage. Tables S2 and S3 contain the CM distance and sex QTL LOD scores of markers within each female and male map, respectively.

\section{Acknowledgements}

We are grateful for the excellent technical support contributed by Lauren Carlson, Jane Petzoldt, Dawn Fishback, and Rebecca Wilk.

\section{Funding}

This work was supported by grants from the United States Department of Agriculture National Institute of Food and Agriculture (USDA-NIFA) \#2015-67009-23957 and \#2018-68005-27925. DGW was supported by USDA NIFA predoctoral fellowship program grant \#2019-67011-29701.

\section{Conflicts of Interest}

The authors have no conflicts of interest to report.

\section{Figures}

Figure 1: Pedigrees of the Salix $F_{1}$ HCP. There were four full-sib families with 94001 as the paternal parent and four full-sib families with 94006 as the maternal parent. Reciprocal crosses were made with male and female S. viminalis and S. suchowensis while S. integra, S. alberti, S. udensis, and S. koriyanagi were crossed only once. P294 was received with the identification of S. alberti.

Figure 2: Results of PC and fastSTRUCTURE analysis of the Salix $F_{1}$ HCP. A: PCA of the parents; B: PCA of the $F_{1}$ and the parents; $C$ : Hierarchical clustering of the parents; D: Distruct plot using fastSTRUCTURE results. In panels A, B, and C: purple - S. purpurea, red - S. viminalis, green - $S$. suchowensis, blue - $S$. udensis, teal $-S$. integra, grey $-S$. koriyanagi, orange - $S$. alberti. Colors in panel $\mathrm{D}$ are based on fastSRUCTURE results, affecting only $S$. alberti. * Received as $S$. alberti. 
362 Figure 3: Linkage maps for each of the parents within the Salix $F_{1} H C P$. Female maps (first and

363 third rows) were constructed using female informative markers ( $A B \times A A)$, while male maps

364 (second and forth rows) were constructed using male informative markers $(A A \times A B)$.

365 Figure 4: Genetic (cM) and Physical (Mb) distances for the maternal parents in the Salix $\mathrm{F}_{1} \mathrm{HCP}$.

366 Each parent has a set of two maps, genetic on the top and physical on the bottom with lines

367 connecting them indicating a marker's relative position in both maps. On the genetic maps,

368 each marker is represented by a grey vertical line while the 1.5 LOD support interval of the QTL

369 is shown with a purple box. On the physical map, grey vertical lines are markers not associated

370 with the QTL, yellow shows markers whose LOD score was above the permuted significance

371 threshold, purple are markers within the 1.5 LOD support interval, and red indicates the $\mathrm{cM}$ and

$372 \mathrm{Mb}$ position of the peak marker. P336's linkage map cM distance was artificially increased for

373 figure clarity.* Received as S. alberti. 


\section{Tables}

Table 1: Sex phenotype statistics for the eight families in the Salix $F_{1}$ HCP.

\begin{tabular}{|c|c|c|c|c|c|c|c|}
\hline Family & $\begin{array}{c}\text { Maternal } \\
\text { Species }\end{array}$ & $\begin{array}{l}\text { Paternal } \\
\text { Species }\end{array}$ & $\mathrm{N}$ & Females & Males & $\begin{array}{c}\text { F:M } \\
\text { Ratio }\end{array}$ & P-Value \\
\hline $13 X-358$ & S. purpurea & S. udensis & 150 & 91 & 59 & $1.6: 1$ & $0.01^{*}$ \\
\hline $10 X-400$ & S. purpurea & S. suchowensis & 88 & 53 & 33 & $1.6: 1$ & $0.03^{*}$ \\
\hline $11 X-407$ & S. purpurea & S. viminalis & 100 & 60 & 40 & $1.5: 1$ & $0.05^{\mathrm{NS}}$ \\
\hline $13 X-438$ & S. purpurea & S. koriyanagi & 150 & 93 & 57 & $1.6: 1$ & $<0.001^{*}$ \\
\hline $12 X-421$ & S. viminalis & S. purpurea & 100 & 53 & 47 & 1.1:1 & $0.55^{\text {NS }}$ \\
\hline $13 X-426$ & S. integra & S. purpurea & 150 & 150 & 0 & $1: 0$ & $<0.001^{*}$ \\
\hline $13 X-440$ & S. suchowensis & S. purpurea & 150 & 91 & 58 & $1.6: 1$ & $0.01^{*}$ \\
\hline $13 X-443$ & S. alberti ${ }^{\ddagger}$ & S. purpurea & 150 & 87 & 62 & $1.4: 1$ & $0.04^{*}$ \\
\hline
\end{tabular}

Chi-square test for sex ratio bias $(p<0.05) ;{ }^{\text {NS }}$ Not significant; ${ }^{\ddagger}$ Received as S. alberti.

Table 2: Sex QTL associated with the SDR within the maternal linkage maps.

\begin{tabular}{ccccccccc}
\hline Family & Maternal Species & Chr & $\begin{array}{r}\text { Peak } \\
\text { (cM) }\end{array}$ & $\begin{array}{c}\text { Peak } \\
\text { LOD }\end{array}$ & $\begin{array}{c}\text { Peak } \\
\text { (Mb) }\end{array}$ & $\begin{array}{c}\text { Low-LSI } \\
\text { (Mb) }\end{array}$ & $\begin{array}{c}\text { High-LSI } \\
\text { (Mb) }\end{array}$ & PVE (\%) \\
\hline $13 X-358$ & S. purpurea & 15 & 85.3 & 29.3 & 8.65 & 4.51 & 9.22 & 73.7 \\
$10 X-400$ & S. purpurea & 15 & 81.1 & 16.1 & 2.91 & 2.61 & 9.92 & 61.8 \\
$11 X-407$ & S. purpurea & 15 & 114.2 & 18.6 & 2.92 & 2.88 & 4.05 & 60.7 \\
$13 X-438$ & S. purpurea & 15 & 70.1 & 35.1 & 7.33 & 2.63 & 8.29 & 71.1 \\
$12 X-421$ & S. viminalis & 15 & 86.0 & 21.1 & 2.92 & 2.95 & 9.36 & 66.0 \\
$13 X-440$ & S. suchowensis & 15 & 78.4 & 26.7 & 2.57 & 2.44 & 9.35 & 74.1 \\
$13 X-443$ & S. alberti $^{\ddagger}$ & 15 & 145.8 & 35.6 & 9.73 & 8.63 & 10.22 & 70.3 \\
\hline
\end{tabular}

LSI: 1.5 LOD Support Interval; PVE: Percent Variation Explained; cM: centiMorgan position within the linkage map; Mb: Physical position based on alignment to $S$. purpurea reference genome. ${ }^{\ddagger}$ Received as S. alberti. 


\section{REFERENCES}

Almeida P., Proux-Wera E., Churcher A., Soler L., Dainat J. et al. 2020. Genome assembly of the basket willow, Salix viminalis, reveals earliest stages of sex chromosome expansion. BMC Biol. 18(1): 78.

Alström-Rapaport C., Lascoux M., Gullberg U. 1997. Sex determination and sex ratio in the dioecious shrub Salix viminalis L. Theor Appl Genet. 94(3-4): 493-497.

Berlin S., Lagercrantz U., von Arnold S., Ost T., Ronnberg-Wastljung A. C. 2010. High-density linkage mapping and evolution of paralogs and orthologs in Salix and Populus. BMC Genomics. 11: 129.

Bradbury P. J., Zhang Z., Kroon D. E., Casstevens T. M., Ramdoss Y. et al. 2007. TASSEL: software for association mapping of complex traits in diverse samples. Bioinformatics. 23(19): 2633-2635.

Broman K. W., Wu H., Sen Ś., Churchill G. A. 2003. R/qtl: QTL mapping in experimental crosses. Bioinformatics. 19(7): 889-890.

Cao Y., Ma C., Chen H., Zhang J., White J. C. et al. 2020. Xylem-based long-distance transport and phloem remobilization of copper in Salix integra Thunb. J Hazard Mater. 392: 122428.

Carlson C. H., Gouker F. E., Crowell C. R., Evans L., DiFazio S. P. et al. 2019. Joint linkage and association mapping of complex traits in shrub willow (Salix purpurea L.). Ann Bot. 124(4): 701-716.

Chen Y., Wang T., Fang L., Li X., Yin T. 2016. Confirmation of single-locus sex determination and female heterogamety in willow based on linkage analysis. PLoS One. 11(2): e0147671.

Clifton-Brown J., Harfouche A., Casler M. D., Dylan Jones H., Macalpine W. J. et al. 2019. Breeding progress and preparedness for mass-scale deployment of perennial lignocellulosic biomass crops switchgrass, miscanthus, willow and poplar. GCB Bioenergy. 11(1): 118-151.

Crowell C. R., Bekauri M. M., Cala A. R., Mcmullen P., Smart L. B. et al. 2020. Differential susceptibility of diverse Salix spp. to Melampsora americana and Melampsora paradoxa. Plant Dis. 104: 29492957. 
Dickmann D. I., Kuzovkina J. 2014. Poplars and willows of the world, with emphasis on silviculturally important species. Poplars and willows: Trees for society and the environment. Rome, Italy: FAO.

Elshire R. J., Glaubitz J. C., Sun Q., Poland J. A., Kawamoto K. et al. 2011. A robust, simple genotyping-bysequencing (GBS) approach for high diversity species. PLoS One. 6(5): e19379.

Fabio E. S., Leary C. J., Smart L. B. 2019. Tolerance of novel inter-specific shrub willow hybrids to water stress. Trees. 33(4): 1015-1026.

Fabio E. S., Smart L. B. 2020. Genetic and environmental influences on first rotation shrub willow (Salix spp.) bark and wood elemental composition. Bioenergy Res. 13(3): 797-809.

Geraldes A., Hefer C. A., Capron A., Kolosova N., Martinez-Nuñez F. et al. 2015. Recent Y chromosome divergence despite ancient origin of dioecy in poplars (Populus). Mol Ecol. 24(13): 3243-3256.

Gouker F. E., Carlson C. H., Zou J., Evans L., Crowell C. R. et al. 2020. Sexual dimorphism and sex ratio bias in the dioecious willow Salix purpurea L. bioRxiv. 2020.2004.2005.026427.

Hallingbäck H. R., Berlin S., Nordh N.-E., Weih M., Rönnberg-Wästljung A.-C. 2019. Genome wide associations of growth, phenology, and plasticity traits in willow [Salix viminalis (L.)]. Front Plant Sci. 10(753).

Hallingbäck H. R., Fogelqvist J., Powers S. J., Turrion-Gomez J., Rossiter R. et al. 2016. Association mapping in Salix viminalis L. (Salicaceae) - identification of candidate genes associated with growth and phenology. GCB Bioenergy. 8(3): 670-685.

Hanley S., Mallott M., Karp A. 2006. Alignment of a Salix linkage map to the Populus genomic sequence reveals macrosynteny between willow and poplar genomes. Tree Genet Genomes. 3(1): 35-48. Hou J., Ye N., Zhang D., Chen Y., Fang L. et al. 2015. Different autosomes evolved into sex chromosomes in the sister genera of Salix and Populus. Scientific Reports. 5(1): 9076. 
Jia H., Wang L., Li J., Sun P., Lu M. et al. 2020. Physiological and metabolic responses of Salix sinopurpurea and Salix suchowensis to drought stress. Trees. 34(2): 563-577.

Kim J., Kim Y., Park J. 2019. Complete chloroplast genome sequence of the Salix koriyanagi Kimura ex Goerz (Salicaceae). Mitochondrial DNA B: Resour. 4(1): 549-550.

Kopp R., Maynard C., Rocha De Niella P., Smart L., Abrahamson L. 2002. Collection and storage of pollen from Salix using organic solvents. Am J Bot. 89: 248-252.

Li H., Durbin R. 2009. Fast and accurate short read alignment with Burrows-Wheeler transform. Bioinformatics. 25(14): 1754-1760.

Li W., Wu H., Li X., Chen Y., Yin T. 2020. Fine mapping of the sex locus in Salix triandra confirms a consistent sex determination mechanism in genus Salix. Hortic Res. 7(1): 64.

Liu J., Yin T., Ye N., Chen Y., Yin T. et al. 2013. Transcriptome analysis of the differentially expressed genes in the male and female shrub willows (Salix suchowensis). PLoS One. 8(4): e60181.

Money D., Migicovsky Z., Gardner K., Myles S. 2017. LinkImputeR: user-guided genotype calling and imputation for non-model organisms. BMC Genomics. 18(1): 523.

Niiyama K. 2008. Coexistence of Salix species in a seasonally flooded habitat. Ecology of Riparian Forests in Japan. Springer. p. 165-174.

Paolucci I., Gaudet M., Jorge V., Beritognolo I., Terzoli S. et al. 2010. Genetic linkage maps of Populus $a l b a$ L. and comparative mapping analysis of sex determination across Populus species. Tree Genet Genomes. 6(6): 863-875.

Park J., Kim Y., Xi H. 2019. The complete chloroplast genome sequence of male individual of Korean endemic willow, Salix koriyanagi Kimura ex Goerz (Salicaceae). Mitochondrial DNA B: Resour. 4(1): 1619-1621.

Pucholt P., Hallingbäck H. R., Berlin S. 2017a. Allelic incompatibility can explain female biased sex ratios in dioecious plants. BMC Genomics. 18(1): 251. 
Pucholt P., Rönnberg-Wästljung A. C., Berlin S. 2015. Single locus sex determination and female heterogamety in the basket willow (Salix viminalis L.). Heredity. 114(6): 575-583.

Pucholt P., Wright A. E., Conze L. L., Mank J. E., Berlin S. 2017b. Recent sex chromosome divergence despite ancient dioecy in the willow Salix viminalis. Mol Biol Evol. 34(8): 1991-2001.

R Core Team. 2020. R: A language and environment for statistical computing. Vienna, Austria: R Foundation for Statistical Computing.

Raj A., Stephens M., Pritchard J. K. 2014. fastSTRUCTURE: variational inference of population structure in large SNP data sets. Genetics. 197(2): 573-589.

Rönnberg-Wästljung A. C., Glynn C., Weih M. 2005. QTL analyses of drought tolerance and growth for a Salix dasyclados $x$ Salix viminalis hybrid in contrasting water regimes. Theor Appl Genet. 110(3): 537-549.

Rönnberg-Wästljung A. C., Samils B., Tsarouhas V., Gullberg U. 2008. Resistance to Melampsora lariciepitea leaf rust in Salix: analyses of quantitative trait loci. J Appl Genet. 49: 321-331.

Rosso L., Facciotto G., Bergante S., Vietto L., Nervo G. 2013. Selection and testing of Populus alba and Salix spp. as bioenergy feedstock: Preliminary results. Appl Energy. 102: 87-92.

Samils B., Rönnberg-Wästljung A.-C., Stenlid J. 2011. QTL mapping of resistance to leaf rust in Salix. Tree Genet Genomes. 7(6): 1219-1235.

Sanderson B. J., Feng G., Hu N., Carlson C. H., Smart L. B. et al. 2021. Sex determination through X-Y heterogamety in Salix nigra. Heredity. 126(4): 630-639.

Slaght J. C., Surmach S. G., Kisleiko A. A. 2018. Ecology and conservation of Blakiston's fish owl in Russia. Biodiversity Conservation Using Umbrella Species. Springer. p. 47-70.

Smart L. B., Volk T., Lin J., Kopp R. F., Phillips I. S. et al. 2005. Genetic improvement of shrub willow (Salix spp.) crops for bioenergy and environmental applications in the United States. Unasylva. 56: 5155. 
Stanton B. J., Serapiglia M. J., Smart L. B. 2014. The domestication and conservation of Populus and Salix genetic resources. Poplars and willows: trees for society and the environment. Wallingford, UK: CAB International. p. 124-199.

Stoof C. R., Richards B. K., Woodbury P. B., Fabio E. S., Brumbach A. R. et al. 2014. Untapped potential: opportunities and challenges for sustainable bioenergy production from marginal lands in the Northeast USA. Bioenergy Res. 8(2): 482-501.

Sulima P., Przyborowski J. A., Kuszewska A., Zaluski D., Jedryczka M. et al. 2017. Identification of quantitative trait loci conditioning the main biomass yield components and resistance to Melampsora spp. in Salix viminalis x Salix schwerinii hybrids. Int J Mol Sci. 18(3).

Taylor J., Butler D. 2017. R Package ASMap: efficient genetic linkage map construction and diagnosis. J Stat Softw. 79(6): 1-29.

Tozawa M., Ueno N., Seiwa K. 2009. Compensatory mechanisms for reproductive costs in the dioecious tree Salix integra. Botany. 87(3): 315-323.

Ueno N., Kanno H., Seiwa K. 2006. Sexual differences in shoot and leaf dynamics in the dioecious tree Salix sachalinensis. Botany. 84(12): 1852-1859.

Ueno N., Seiwa K. 2003. Gender-specific shoot structure and functions in relation to habitat conditions in a dioecious tree, Salix sachalinensis. J For Res. 8(1): 0009-0016.

Wei S., Yang Y., Yin T. 2020. The chromosome-scale assembly of the willow genome provides insight into Salicaceae genome evolution. Hortic Res. 7(1): 45.

Yang G., Xu Q., Li W., Ling J., Li X. et al. 2020a. Sex-related differences in growth, herbivory, and defense of two Salix species. Forests. 11(4): 450.

Yang W., Wang D., Li Y., Zhang Z., Tong S. et al. 2021. A general model to explain repeated turnovers of sex determination in the Salicaceae. Mol Biol Evol. 38(3): 968-980. 
497 Yang W., Wang Y., Liu D., Hussain B., Ding Z. et al. 2020b. Interactions between cadmium and zinc in uptake, accumulation and bioavailability for Salix integra with respect to phytoremediation. Int J Phytoremediation. 22(6): 628-637.

500 Zhou R., Macaya-Sanz D., Carlson C. H., Schmutz J., Jenkins J. W. et al. 2020. A willow sex chromosome reveals convergent evolution of complex palindromic repeats. Genome Biol. 21(1): 38.

502 Zhou R., Macaya-Sanz D., Rodgers-Melnick E., Carlson C. H., Gouker F. E. et al. 2018. Characterization of 


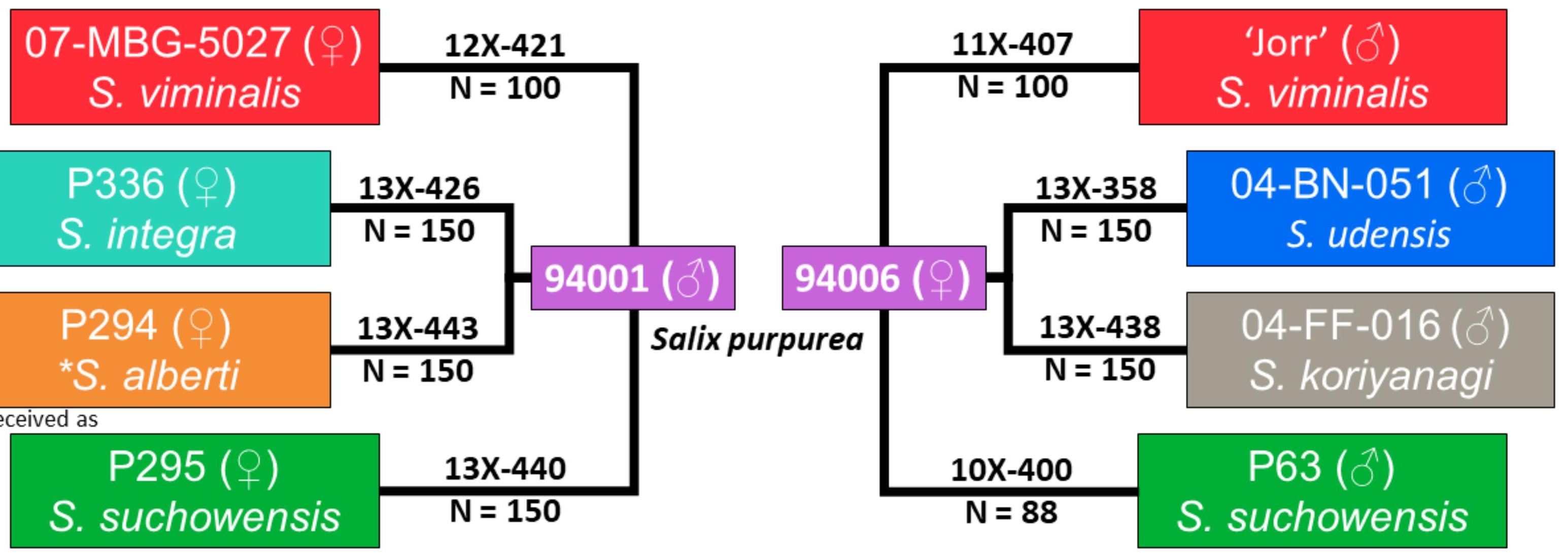




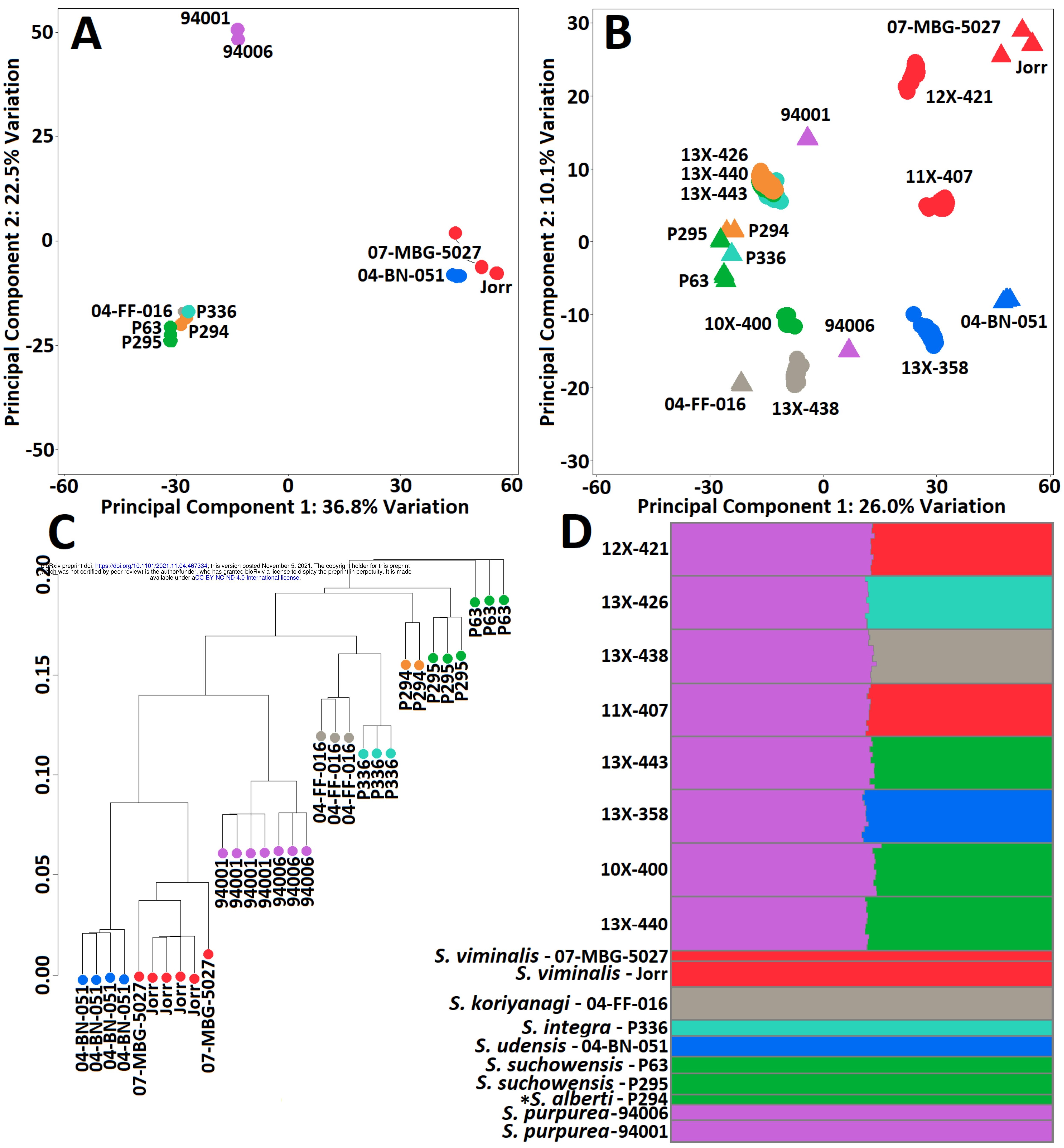




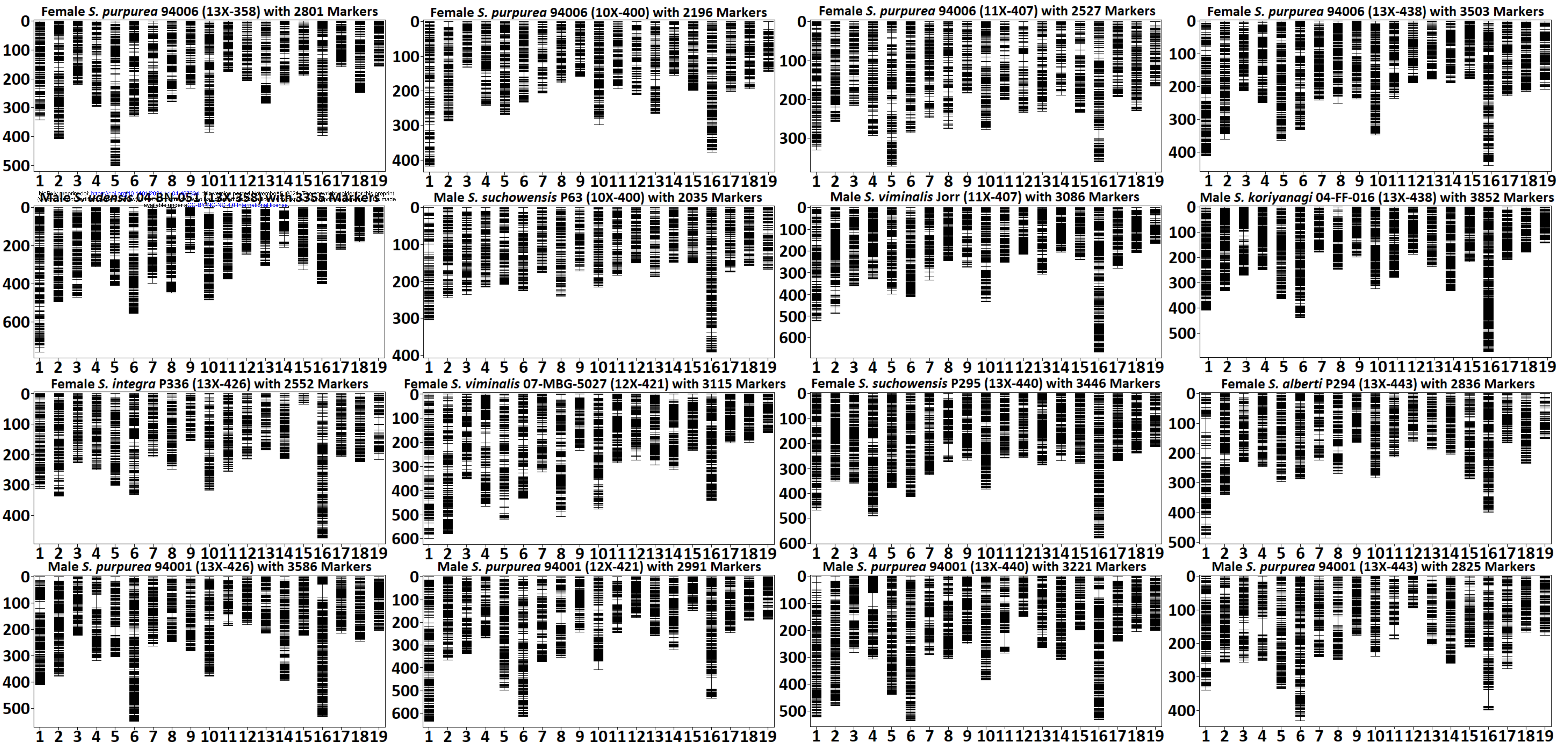


Genetic Distance (cM)

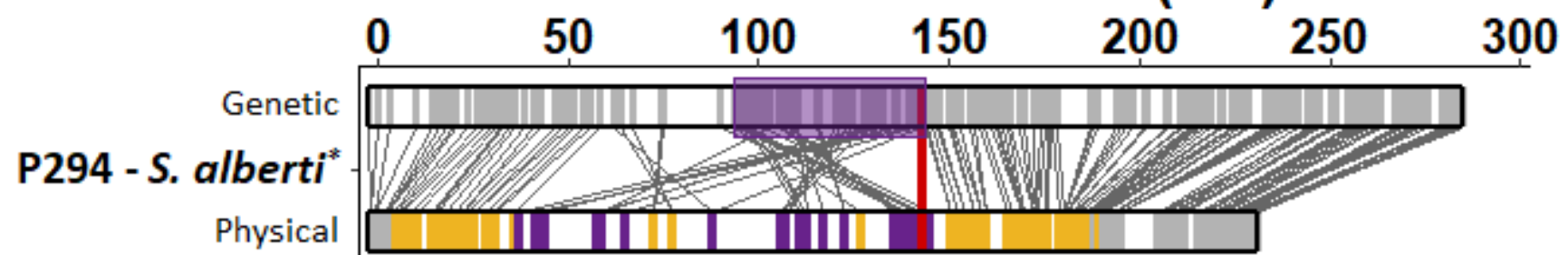

P295 - S. suchowensis

Physical

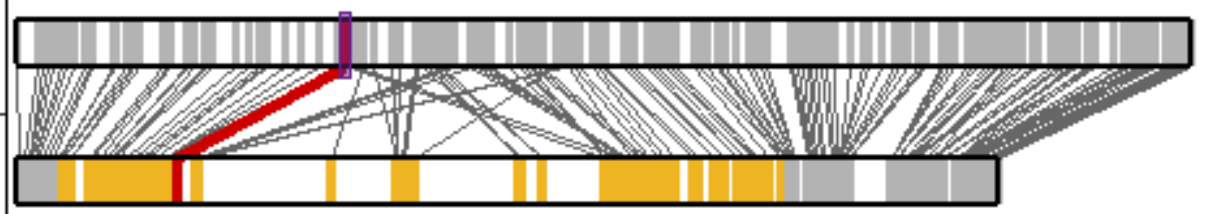

P336 - S. integra

Physical

07-MBG-5027 - S. viminalis

Physical

Genetic

94006 (13X-438) - S. purpurea

Physical

94006 (11X-407) - S. purpurea

Physical
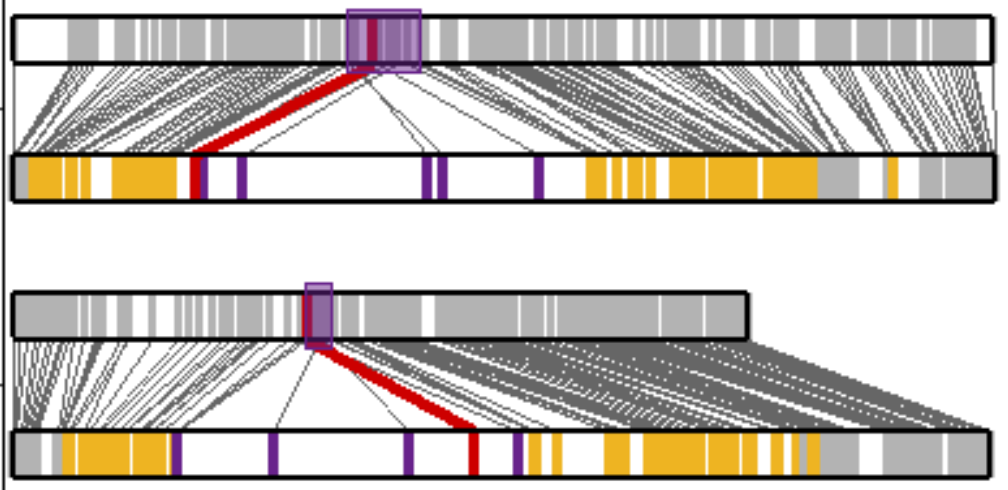

Genetic

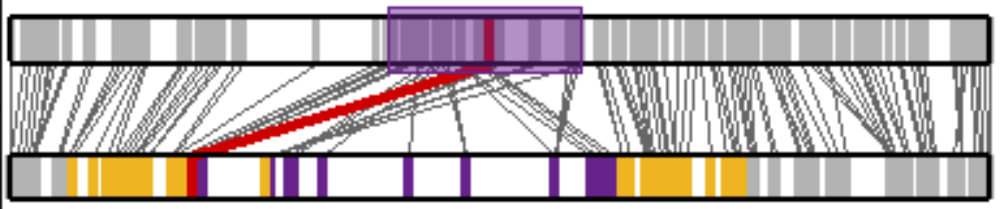

94006 (10X-400) - S. purpurea

Physical

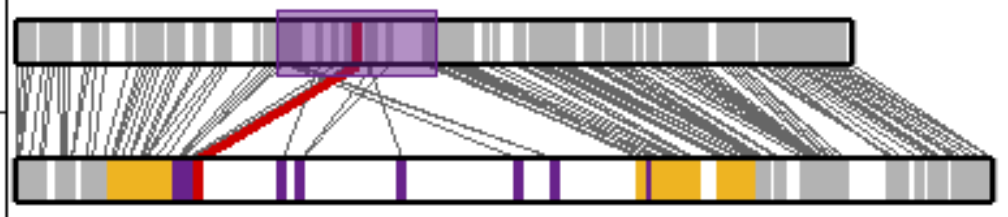

94006 (13X-358) - S. purpurea

Physical

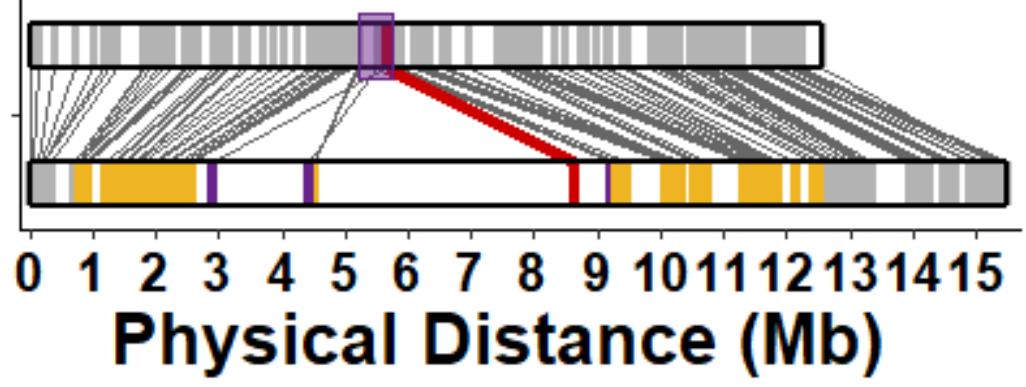

